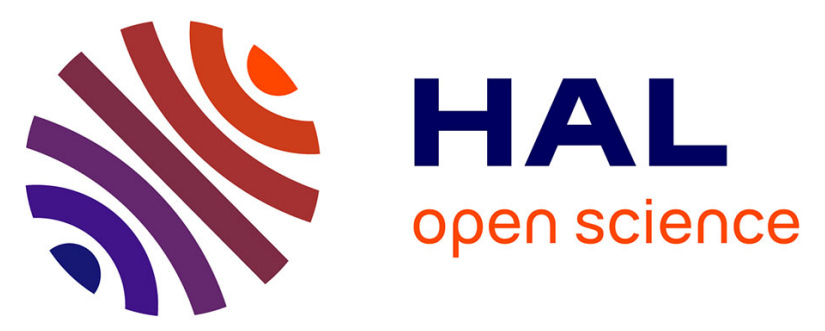

\title{
Sliding planar anchoring and viscous surface torque in a cholesteric liquid crystal
}

Patrick Oswald, Alain Dequidt, Andrzej Zywocinski

\section{To cite this version:}

Patrick Oswald, Alain Dequidt, Andrzej Zywocinski. Sliding planar anchoring and viscous surface torque in a cholesteric liquid crystal. Physical Review E: Statistical, Nonlinear, and Soft Matter Physics, 2008, 77 (6), pp.061703. 10.1103/PhysRevE.77.061703 . ensl-00351712

\section{HAL Id: ensl-00351712}

\section{https://hal-ens-lyon.archives-ouvertes.fr/ensl-00351712}

Submitted on 10 Jan 2009

HAL is a multi-disciplinary open access archive for the deposit and dissemination of scientific research documents, whether they are published or not. The documents may come from teaching and research institutions in France or abroad, or from public or private research centers.
L'archive ouverte pluridisciplinaire HAL, est destinée au dépôt et à la diffusion de documents scientifiques de niveau recherche, publiés ou non, émanant des établissements d'enseignement et de recherche français ou étrangers, des laboratoires publics ou privés. 


\title{
Manuscript Title: Sliding planar anchoring and viscous surface torque in a cholesteric liquid crystal
}

\author{
Patrick Oswald, ${ }^{*}$ Alain Dequidt, and Andrzej Żywociński ${ }^{\dagger}$ \\ Université de Lyon, Laboratoire de Physique, \\ École Normale Supérieure de Lyon, CNRS, \\ 46 Allée d'Italie, 69364 Lyon, France.
}

(Dated: April 2, 2008)

\begin{abstract}
We propose a new surface treatment allowing to obtain a sliding planar anchoring of nematic (or cholesteric) liquid crystals. It consists of depositing a thin layer of the polymercaptan hardener of an epoxy resin on an isotropic substrate (bare or ITO-coated glass plates). Microscopic observations of defect annihilations and capacitance measurements show that the molecules align parallel to the surface and slide viscously on it when they change orientation, which implies a zero (or extremely small) azimuthal anchoring energy. By contrast, the zenithal anchoring energy $W_{\theta}$ is found to be larger than $3 \times 10^{-5} \mathrm{~J} / \mathrm{m}^{2}$. We also measured the liquid crystal rotational surface viscosity $\gamma_{S}$ by a new thermo-optical method using the large temperature variation of the pitch of a compensated cholesteric mixture. We found that the sliding length $\gamma_{S} / \gamma_{1}$ (where $\gamma_{1}$ is the bulk rotational viscosity) is very large in comparison with the length of a liquid crystal molecule. This result is explained by a simple model which takes into account the diffusion of the liquid crystal within the polymer layer.

PACS numbers: 61.30.Hn, 42.70.Df
\end{abstract}

\section{INTRODUCTION}

In a nematic liquid crystal, the rod-like molecules tend to have the same direction, their centers of mass being distributed randomly. Order is purely orientational and characterized by a unit vector $\vec{n}$ parallel to the average orientation of the molecules (with $\vec{n} \Leftrightarrow-\vec{n}$ ). If one adds a chiral impurity to a nematic phase, one usually obtains a cholesteric phase in which the director turns around a space direction called the helical axis [1]. The distance over which the director rotates by $2 \pi$ is called the cholesteric pitch. As we shall see later, the pitch can sometimes strongly depend on temperature. One important property of these materials is their ability to be oriented by the surfaces in contact with them. In particular, considerable effort has been expended in the past to find surface treatments allowing for strong planar or homeotropic anchoring of the molecules. In the first case, the molecules orient parallel to the surface, whereas in the second case, the molecules are perpendicular to it. These two anchorings are characterized by a single direction named "surface easy axis" $\vec{n}_{s}$. "Strong" means that the molecules shift very little from $\vec{n}_{s}$ when the director field is deformed in the bulk under the action of an external mechanical, electric or magnetic torque. In this "geometric" limit, the surfaces play a passive role as they only fix the orientation of the molecules in contact with them. These boundary conditions were used in most experiments till recently and continue to play a major role in display applications. In particular, the strong planar anchoring is widely used in making the twisted or super-twisted

\footnotetext{
*Electronic address: patrick.oswald@ens-lyon.fr

†Permanent address: Department III, Institute of Physical Chemistry, Polish Academy of Sciences, Kasprzaka 44/52, 01-224 Warsaw, Poland.
} 
nematic display.

Other types of anchoring exist [2]. For instance, oblique monostable or bistable anchorings have been observed on evaporated $\mathrm{SiO}$ layers [3] or on cleaved surfaces of crystals such as mica [4] with the possibility of passing from one to another by changing a physical parameter such as the temperature or the humidity (for a review about anchoring transitions, see chapter V of Ref. [1]). More recently, planar and oblique azimuthally degenerate anchorings have also been reported in the literature. Different techniques have been proposed to reach such boundary conditions. One of them has consisted of adding to the liquid crystal a small amount of oligomeric molecules [5]. This method was shown to lead to very small zenithal (out of substrate plane) and azimuthal (in plane) anchoring energies with the liquid crystal 5CB. Another technique consists of depositing a "thick" layer (more than 100 $\AA$, typically) of a surfactant belonging to the family of the organofunctional silanes. One of them, the (3-glycidoxypropyl) trimethoxysilane (also called 3-GPS or GLYMO) gives a planar degenerated anchoring with the liquid crystal 5CB [6]. One can also graft on the surface highly mobile polymer chains. For instance, grafted polystyrene was shown to lead to a conicallydegenerated anchoring of the nematic 5CB with a pretty small zenithal anchoring energy [7]. Thin layers of the photopolymer NOA60 was also used recently to produce a planar anchoring with an ultraweak azimuthal anchoring [8]. These new anchorings are interesting for two reasons:

-first, they open the possibility of making new and more performing displays in which the surface now plays an active role, the anchoring conditions changing when switching between two states of the display [9-11];

-second, they allow to perform new experiments in the field of fundamental physics.

A good example was the first direct observation of the smectic blocks in a $\mathrm{TGB}_{\mathrm{A}}$ phase. In this experiment, the GLYMO surface treatment was used [12]. Another example was the experimental evidence of backflow effects during the collapse of two disclination lines [13]. In this case, the surfaces were treated with the UV glue NOA60. It must be noted that in this experiment, an electric field was used to move the lines, which shows that the anchoring was not completely sliding.

In the present paper, we describe a new surface treatment we recently used to evidence the thermomechanical Lehmann effect in cholesteric liquid crystal $[14,15]$. This surface treatment turned out to give planar and sliding anchoring, which was essential for observing the Lehmann effect. In our case, it consisted of spin coating a thin layer of the polymercaptan hardener of an epoxy resin (Structuralit 7).

The goal of the paper is to establish the unique properties of this new surface treatment as it gives a planar alignment of the molecules on the surface with a strong zenithal anchoring energy, while allowing the molecules to rotate viscously on the surface (which implies a zero or extremely small azimuthal anchoring energy). To show these results we conducted microscopic observations of defect annihilations and capacitance measurements. We also developed a new thermo-optical method to determine the liquid crystal surface viscosity $\gamma_{S}$, a quantity difficult to measure and very little documented in the literature in comparison with the anchoring energy.

The plan of the article is the following. In section II, we describe the sample preparation and we show from microscopic observation of the optical texture and from capacitance measurements that the anchoring of the molecules on the polymercaptan is planar and sliding. In section III, we measure the bulk rotational viscosity $\gamma_{1}$ and show that the presence of the polymeric layer does not change its value. In section IV, we show how to measure the surface viscosity $\gamma_{S}$ of the liquid crystal by measuring the variations of the optical transmittance of samples of different thicknesses during temperature ramps. The measured value of $\gamma_{S}$ is discussed within a simple model in section $\mathrm{V}$, while in section VI, we discuss the problem of the annihilation of two $\pm 1 / 2$ disclination lines. Finally, conclusions are drawn in section VII. 


\section{SAMPLE PREPARATION AND CAPACITANCE MEASUREMENTS}

The liquid crystal chosen was a mixture of 4-n-octyloxy-4'-cyanobiphenyl (8OCB from Synthon Chemicals GmbH $\& \mathrm{Co}$ ) and of cholesteryl chloride ( $\mathrm{CC}$ from Aldrich) in proportion 1:1 in weight with a compensation point at $T_{c}=59^{\circ} \mathrm{C}$

and a clearing point at $66.5^{\circ} \mathrm{C}$. We recall that at $T_{c}$, the equilibrium twist vanishes and changes sign. The phase has thus a nematic-like structure at this particular temperature. The 8OCB was purified by one of us (AZ) and the CC was used without further purification. The mixture was filtered in the cholesteric phase through $0.2 \mu \mathrm{m}$ nylon filter to remove dust particles.

The samples were prepared between two glass plates treated either for strong planar unidirectional anchoring or for planar sliding anchoring.

The first anchoring is classical and was obtained by covering the surface with a rubbed polyimide layer baked at $300^{\circ} \mathrm{C}$ during 2 hours (ZLI 2650 from Merck). To realize the second anchoring, we treated the surface with the polymercaptan hardener of an epoxy glue (Structalit 7 from Eleco). This polymer was first dissolved in a ketone, the 2 -butanone ( $5 \%$ in mass of hardener). The obtained solution was then filtered through $0.2 \mu \mathrm{m}$ PTFE membrane to eliminate dust particles and spread by spin-coating on the plate at $500 \mathrm{rpm}$ for $1 \mathrm{mn}$. In this way, the polymercaptan harderner formed a homogeneous thin layer wetting perfectly the surface (up to $100^{\circ} \mathrm{C}$, at least). Its thickness was measured by Michelson interferometry and was found to be of the order of $0.2 \mu \mathrm{m}$. In addition, the layer was held at $60^{\circ} \mathrm{C}$ for one hour under vacuum in order to completely evaporate the ketone. This precaution is important to not pollute the liquid crystal [16].

Finally, nickel and tungsten wires of calibrated diameters were used as spacers to fix the sample thickness.

In order to prove that this new surface treatment gives a sliding planar anchoring, we performed the two following experiments.

In the first one, we made a $10 \mu \mathrm{m}$-thick sample with the two glass plates treated with the polymercaptan hardener. The liquid crystal was first introduced by capillarity in the isotropic phase and then cooled down into the cholesteric phase. As a result we observed the formation of a "Schlieren" texture containing a large quantity of $\pm 1 / 2$ disclination lines perpendicular to the surfaces (Fig. 1a). This observation clearly shows that the anchoring is planar (and not oblique) and azimuthally degenerate since it is the only orientation allowing such defects. Note that \pm 1 defects are also present, but they are much less numerous that the previous because they cost more elastic energy [1]. In addition, we observed that isolated pairs of opposite defects were able to spontaneously annihilate (Fig. 1b). This is a clear indication that the anchoring is sliding, as otherwise the defects could not move. This is compatible with a zero (or extremely small) azimuthal anchoring energy and the absence of memory effects, at least over the time scale of our experiments, i.e. 2-3 days. In this respect, our anchoring seems to be more sliding than that reported in Ref. [5] or in Ref. [13] (in this work, in particular, where the UV glue NOA60 was used, it was necessary to apply a small horizontal electric field to observe the annihilation of the defects). We also observed that defects of positive strength moved faster than defects of negative strength, in agreement with previous experiments [13, 17] and theoretical works [18-20]. Finally, we emphasize that the annihilation is a slow process in spite of the sliding anchoring as long as the distance between the two defects is larger than a few tens of a micrometer. This point will be discussed in detail in Section VI.

To confirm that the anchoring was planar, we performed capacitance measurements. More precisely, we made two $10 \mu \mathrm{m}$-thick samples, the first one treated for strong planar anchoring on one plate and for sliding anchoring on the opposite one (called sample of type I in the following), whereas the second sample was treated for strong planar (and parallel) anchoring on both sides (called sample of type II in the following). In both cases, the glass plates were 

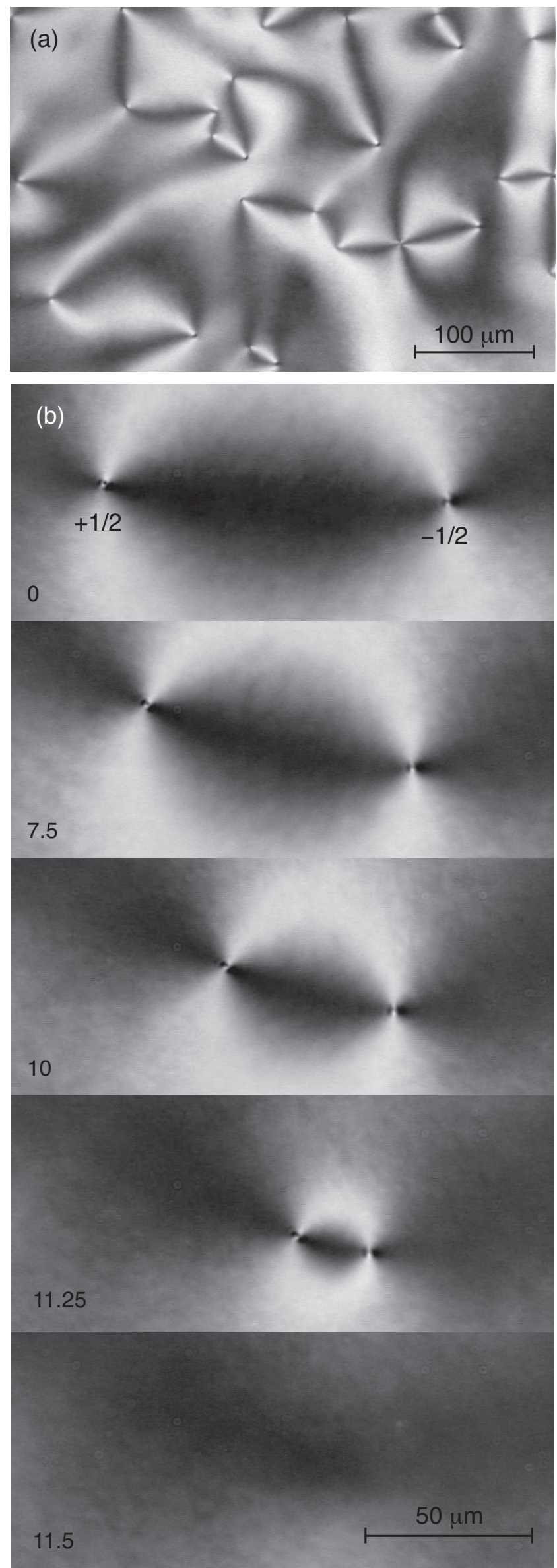

FIG. 1: a) Texture observed at the compensation temperature after a quench from the isotropic liquid. b)Sequence of photographs showing the spontaneous collapse at of two $\pm 1 / 2$ disclination lines. Note the $+1 / 2$ defect moves faster than the $-1 / 2$ defect. Time is given in min at the bottom left hand side of the photographs. $d=10 \mu \mathrm{m}$. Crossed polarizers. 
coated with ITO layers to impose the electric field. In Fig. 2, we show the capacitances of the two cells measured at the compensation temperature as a function of the applied voltage at frequency $f=10 \mathrm{kHz}$. In the figure, the capacitances $C$ are normalized to their values $C_{\perp}$ measured below the threshold of Fredericksz instability $V_{c}$ (of the order of $0.92 \mathrm{Vrms}$ in both samples, which proves, by the way, that the presence of polymercaptan hardener does not change the value of the splay constant $K_{1}$ as well). Within experimental errors, the two curves superimpose. In addition, measurements of the capacitances of the cells before and after filling with the liquid crystal led to similar values (within $1 \%$ ) of $\varepsilon_{\perp}$ (4.52 and 4.50, respectively), in very good agreement with the value given previously (4.5 \pm 0.2 $[14,15])$.

From this experiment, we concluded that the anchoring of the liquid crystal on the polymercaptan hardener is planar and rather strong zenithally since it behaves like the polyimide layer up to an electric field of $2 \mathrm{~V} / \mu \mathrm{m}$. More precisely, we estimated a lower bound for the zenithal anchoring energy $W_{\theta}$ by noting that at electric field $E_{\max }=2 \mathrm{~V} / \mu \mathrm{m}$, the liquid crystal was not fully realigned by the field close to plate treated with the polymercaptan hardener. As this phenomenon occurs for electric fields larger than the saturation field $E_{s}=W_{\theta} / \sqrt{\varepsilon_{0} \varepsilon_{a} K}$ [21] (with $\epsilon_{a}$ the dielectric anisotropy and $K$ the average of the splay and bend elastic constants), we deduced that $W_{\theta}>E_{\text {max }} \sqrt{\varepsilon_{0} \varepsilon_{a} K}$ since $E_{\max }$ was less than $E_{l}$ experimentally. That gives $W_{\theta}>3 \times 10^{-5} \mathrm{~J} / \mathrm{m}^{2}$ knowing that $\varepsilon_{a}=5$ and $K=4.6 \times 10^{-12} \mathrm{~N}$ $[14,15]$.

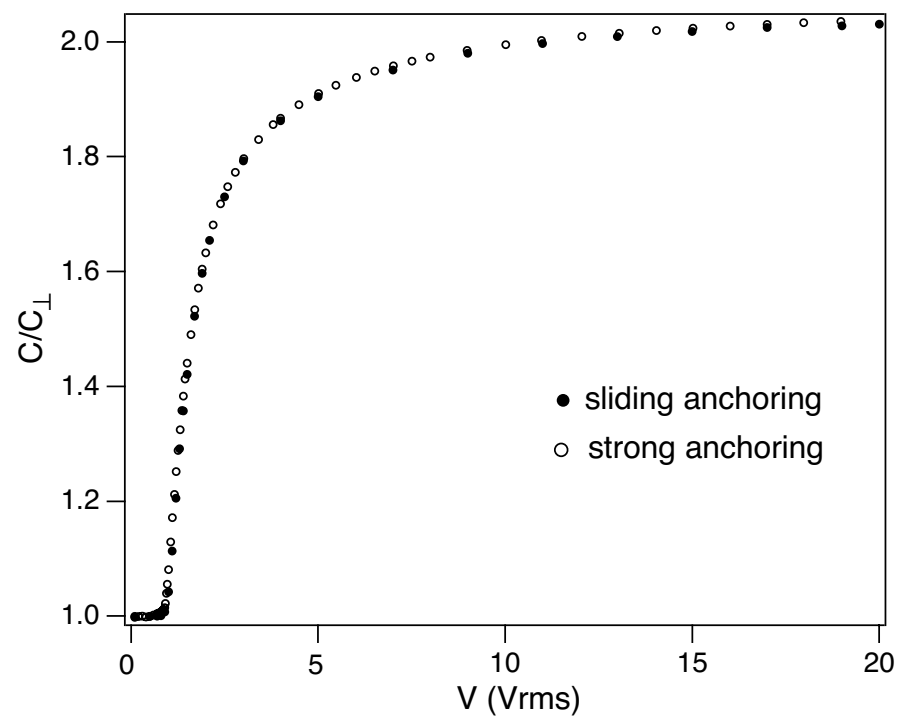

FIG. 2: Capacitance as a function of the applied voltage $(f=10 \mathrm{kHz})$ measured at the compensation temperature $T_{c}$ in a standard planar sample and in a sample treated on one plate for sliding anchoring. The curves superpose within experimental errors.

\section{ROTATIONAL VISCOSITY $\gamma_{1}$}

In practice, the melting temperature of the cholesteric phase is lower by typically $1^{\circ} \mathrm{C}$ in samples treated for sliding anchoring. In contrast, the compensation temperature does not change within $\pm 0.2^{\circ} \mathrm{C}$. This suggests that a small amount of the polymercaptan hardener dissolves in the liquid crystal. In the following section, we shall need the value of the bulk rotational viscosity $\gamma_{1}$. For this reason and to investigate the influence of the dissolved polymer, we measured $\gamma_{1}$ in planar samples of the two types (as defined in the previous section) at the compensation 
temperature. The method used was classical and consisted of first destabilizing the sample with a large electric field (20 Vrms, $f=10 \mathrm{kHz}$ ) and then, of observing the relaxation of the distorted director field after the electric field was removed. In practice, the sample is placed between crossed polarizers at $45^{\circ}$ of the anchoring direction and its optical transmittance is measured by a photodiode connected to a memory oscilloscope. Curves obtained with samples of types I and II are shown in Fig. 3. To fit these curves, we took advantage that we previously measured the elastic constants, the dielectric constants and the optical indices of the liquid crystal at the compensation point: $K_{1}=3.4 \times 10^{-12} \mathrm{~N}, K_{3}=5.9 \times 10^{-12} \mathrm{~N}, \varepsilon_{\|}=9.4, \varepsilon_{\perp}=4.5, n_{o}=1.55$ and $n_{e}=1.64$ [14, 15]. Using these data, it was possible to solve numerically the governing equations for the director field (in anisotropic elasticity) and to then calculate the optical transmittance. We took as fit parameters the sample thickness $d$ and the classical relaxation time $\tau=\frac{\gamma_{1} d^{2}}{\pi^{2} K_{1}}$. Two fits are shown respectively in Fig. 3(a) for a type I sample and in Fig. 3(b) for a type II sample. In the first case, we found $d=29.2 \mu \mathrm{m}$ and $\tau=1.91 \mathrm{~s}$, while $d=30.5 \mu \mathrm{m}$ and $\tau=2.07 \mathrm{~s}$ in the second sample. In these two examples, the fitted thickness was very close to the nominal one $(30 \mu \mathrm{m})$. We thus calculated $\gamma_{1}$ by taking the value of the thickness given by the fit and $K_{1}=3.4 \times 10^{-12} \mathrm{~N}[14,15]$. This procedure gave the same value in type I and type II samples: $\gamma_{1}=0.075 \pm 0.009$ Pa.s. We performed similar measurements in $20 \mu$ m-thick commercial type II cells (from Instec, Inc) and found again a similar value within experimental errors.
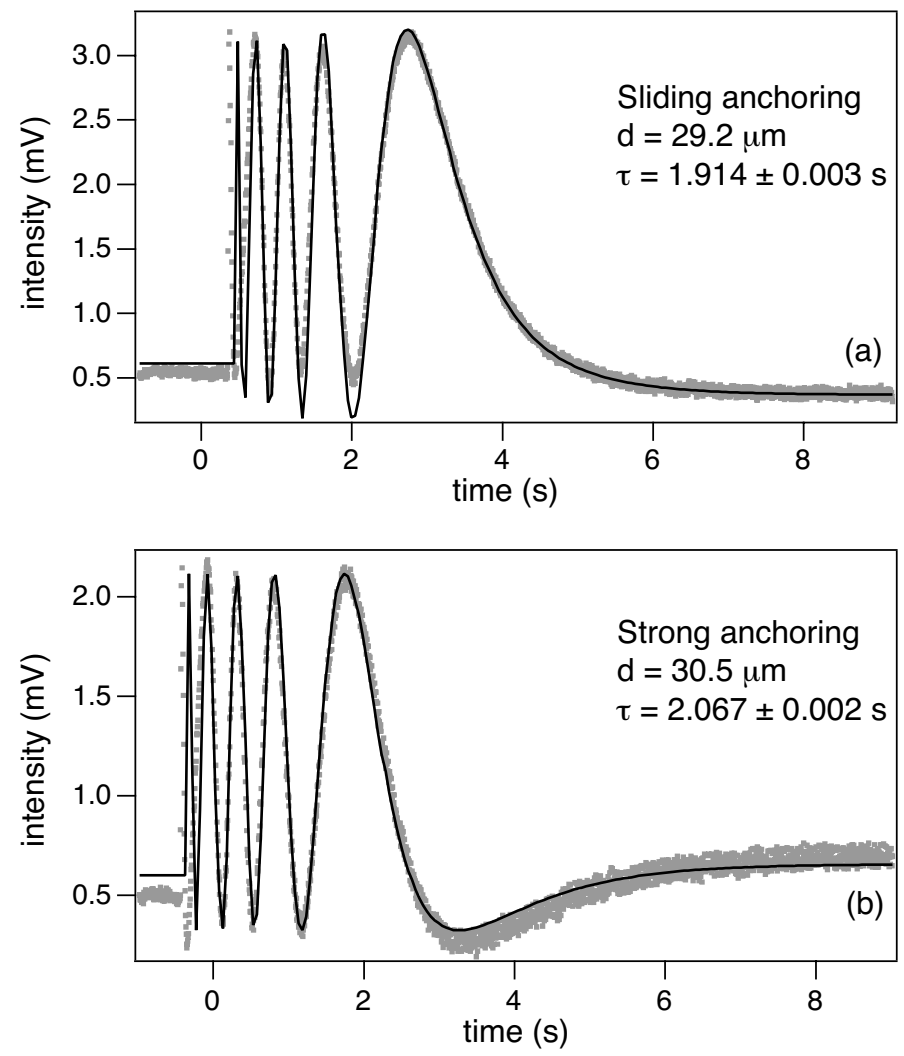

FIG. 3: Optical transmittance measured at $T=T_{c}$ between crossed polarizers at $45^{\circ}$ of the anchoring direction as a function of time after switching off the voltage. Points are experimental and correspond to several curves measured at different places in the sample. The solid line is the best fit to the numerical model. a) Type I sample; b) Type II sample.

In conclusion, the rotational viscosity $\gamma_{1}$ does not chan-ge in a measurable way in the presence of the polymercaptan hardener. This result agrees with previous conclusions of Jăkli et al [22] about the role in the value of $\gamma_{1}$ of a polymer dissolved in the liquid crystal. In the following, we shall take $\gamma_{1}=0.075 \pm 0.009$ Pa.s. 


\section{SURFACE VISCOSITY $\gamma_{S}$}

In practice, the director does not rotate freely at the surface of the liquid polymercaptan layer, but experiences a viscous surface torque which can be written in the usual form [23]

$$
\vec{\Gamma}_{S}=-\gamma_{S} \vec{n} \times \frac{\partial \vec{n}}{\partial t}
$$

The surface viscosity $\gamma_{S}$ has the dimension of a bulk viscosity times a length. This length $l_{S}$ characterizes the distance over which the liquid crystal interacts with the polymer layer. It will be discussed in Section 5 . In the next three subsections, we successively describe the principle of the experiment, the technique of measurements and the experimental results.

\section{A. Principle of the experiment}

The experiment consisted of measuring the optical transmittance in monochromatic light $(\lambda=546 \mathrm{~nm})$ of a type I sample as a function of the temperature when it was changed at a constant rate. The sample was placed between crossed polarizer and analyzer with the polarizer parallel to the anchoring direction imposed on the lower glass plate. Due to the sliding anchoring on the upper plate, the transmittance changed with the temperature and vanished (by assuming an adiabatic rotation of the plane of polarization of the light) when the director on this surface was perpendicular to the analyzer. We checked numerically that this optical condition was fulfilled at a temperature $T_{0}$ (close to $T_{c}$ ) being a function of the imposed temperature $\operatorname{ramp} r=d T / d t$ (with $T_{0} \rightarrow T_{c}$ when $r \rightarrow 0$ ). The experiment thus consisted of measuring $T_{0}(r)$ in samples of different thicknesses $d$. In the following, we show that the derivative $d T_{0} / d r$ is a well-defined function of $d, \gamma_{1}$ and $\gamma_{S}$.

In order to calculate this quantity, we first recall the governing equations of the problem. Let $\varphi$ be the angle between the director and the anchoring direction on the bottom plate. Because the director remains parallel to the glass plates, it is only function of $z$ and $t$ (with the $\mathrm{z}$ axis perpendicular to the plates). If, in addition, the temperature and thus, the equilibrium twist $q$, can be supposed independent of $z$, an assumption we shall justify in the Appendix, then the bulk torque equation simply reads

$$
\gamma_{1} \frac{\partial \varphi}{\partial t}=K_{2} \frac{\partial^{2} \varphi}{\partial z^{2}}
$$

At the bottom plate, the director orientation is fixed, so that

$$
\varphi=0 \text { at } z=0
$$

while at the top plate, the anchoring is sliding, imposing

$$
\gamma_{S} \frac{\partial \varphi}{\partial t}=-K_{2}\left[\frac{\partial \varphi}{\partial z}-q[T(t)]\right] \text { at } z=d
$$

where $q[T(t)]$ is the equilibrium twist of the cholesteric.

Experimentally, we impose a constant temperature ramp $r=d T / d t$, so that

$$
T(t)=T_{c}+r t
$$

In addition, we know from previous measurements that $q$ is, to a very good approximation, proportional to $T-T_{c}$ in the vicinity of the compensation point $[14,15]$. This allows us to write that

$$
q d=\omega t \text { with } \omega=r d \frac{d q}{d T}
$$


Let us now define the angle $\delta(z, t)=\varphi(z, t)-q z$ characterizing the shift to the perfect helix. According to Eqs. 1 and 6 , this angle must satisfy the following equation

$$
\gamma_{1} \frac{\partial \delta}{\partial t}=K_{2} \frac{\partial^{2} \delta}{\partial z^{2}}-\gamma_{1} \omega \frac{z}{d}
$$

In the stationary regime ( $\delta$ independent of $t$ ), this equation has the general solution:

$$
\delta=\frac{\omega}{3 \omega_{b}}\left(\frac{z}{d}\right)^{3}+a z+b
$$

where $\omega_{b}=\frac{2 K_{2}}{\gamma_{1} d^{2}}$ is a bulk relaxation rate.

Constants $b$ and $a$ are respectively given by the boundary conditions 3 and 4 :

$$
b=0 \text { and } a=-\frac{\omega}{d}\left(\frac{1}{\omega_{b}}+\frac{1}{\omega_{S}}\right)
$$

where $\omega_{S}=\frac{K_{2}}{\gamma_{S} d}$ is a surface relaxation rate. Coming back to angle $\varphi$, the general solution reads in the stationary regime:

$$
\varphi=\frac{\omega}{3 \omega_{b}}\left(\frac{z}{d}\right)^{3}+\omega\left[t-\left(\frac{1}{\omega_{b}}+\frac{1}{\omega_{S}}\right)\right]
$$

Close to $T_{c}$, the optical transmittance vanishes when $\varphi(d, t)=0$. This condition is fulfilled at time $t_{0}=\frac{1}{\omega_{S}}+\frac{2}{3 \omega_{b}}$ corresponding to temperature $T_{0}=T_{c}+r\left(\frac{1}{\omega_{S}}+\frac{2}{3 \omega_{b}}\right)$ according to Eq. 6 .

Finally we obtain the researched quantity:

$$
\frac{d T_{0}}{d r}=\frac{1}{\omega_{S}}+\frac{2}{3 \omega_{b}}=\frac{\gamma_{S}}{K_{2}} d+\frac{\gamma_{1}}{3 K_{2}} d^{2}
$$

In the following section, we show how to measure this quantity.

\section{B. Experiment}

We used a Mettler oven to impose temperature ramps to the samples. The oven was placed on the stage of a polarizing Leica microscope and the optical transmittance was measured with a photodiode. To measure the temperature of the sample we used a local sensor consisting of a $10 \mu \mathrm{m}$ in diameter tungsten wire placed between the two glass plates a few mm apart from the zone of interest. Its resistance was measured via a four-wire method with a Keihtley 2001 multimeter interfaced with a PC and was found to linearly depend on the temperature. The temperature stability of this home-made sensor was excellent as no visible drift was observed over many days. An example of a measured temperature profile is shown in Fig. 4 . For slow ramps (inferior to $3^{\circ} \mathrm{C} / \mathrm{min}$ in absolute value), we found very good agreement between nominal and measured values. For faster ramps, small differences were observed. For this reason, we measured systematically the temperature ramp to minimize errors.

An attentive reader could object that the temperature measured in this way could be different from the temperature in the middle of the sample where optical measurements were performed. For this reason, we realized a dummy sample with one wire on the side (as in usual samples) and another in the middle. We then recorded as a function of time the temperatures given by the two wires with a Keihtley 2001 multimeter equipped with a scanner card. In Fig. 5 we plotted the temperature of the side sensor as a function of the temperature of the middle sensor. Four curves are shown corresponding to two pairs of ramps of opposite signs. The two curves corresponding to the $\pm 2^{\circ} \mathrm{C} / \mathrm{min} \mathrm{ramps}$ superimpose perfectly (within the experimental noise); by contrast, a systematic small shift (certainly depending on the thermal contact between the sample and the oven, but always within $\pm 0.1^{\circ}$ ) was often detected for the fastest 


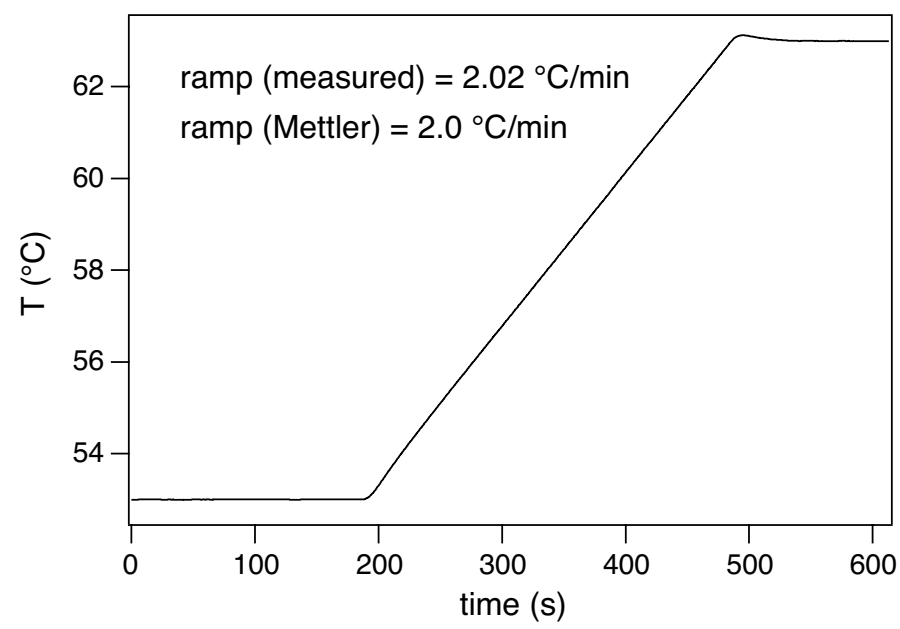

FIG. 4: Temperature profile measured in situ with the tungsten wire. The measured value of the temperature ramp is here slightly larger than its nominal value given by the Mettler oven. This difference increases when the ramp is faster, but never exceeds $5 \%$.

ramps $\left( \pm 7^{\circ} \mathrm{C} / \mathrm{min}\right)$. This test proved that our sensor did give the temperature of the sample at the place where measurements were performed with a negligible error for slow ramps and an error that does not exceed $\pm 0.1^{\circ} \mathrm{C}$ for the fastest ramps.

The next step was to determine for each sample its compensation temperature. The used method consisted of recording its optical transmittance as a function of temperature under a very slow temperature ramp (typically $\left.0.2^{\circ} \mathrm{C} / \mathrm{min}\right)$. Note we used two Keihtley multimeters interfaced with a PC to record the photodiode voltage (proportional to the optical transmittance) and the temperature given by the tungsten wire. At this speed, the cholesteric phase may be considered at equilibrium during the ramp and its optical transmittance is given by the following formula:

$$
\begin{array}{r}
\frac{I}{I_{0}}=\frac{1}{2}-\cos (2 q d) \frac{\kappa^{2}+\cos \left(2 q d \sqrt{1+\kappa^{2}}\right)}{2\left(1+\kappa^{2}\right)} \\
-\sin (2 q d) \frac{\sin \left(2 q d \sqrt{1+\kappa^{2}}\right)}{2 \sqrt{1+\kappa^{2}}}
\end{array}
$$

with $\kappa=\frac{\pi \Delta n}{q \lambda}(\lambda=546 \mathrm{~nm})$.

A typical experimental curve is shown in Fig 6 . To fit it to the previous law, we chose as free parameters the compensation temperature $T_{c}$ (knowing that the equilibrium twist is given by $q\left(\mu \mathrm{m}^{-1}\right)=0.1365\left(T-T_{c}\right)+0.00284(T-$ $\left.T_{c}\right)^{2}$, with the temperatures in ${ }^{\circ} \mathrm{C}[14,15]$ ), the sample thickness $d$, the intensity $I_{0}$ and the birefringence (which we took in the form $\Delta n=a-b\left(T-T_{c}\right)$ ). This led to $T_{c}=58.9^{\circ} \mathrm{C}, I_{0}=16.9 \mathrm{mV}, d=23.8 \mu \mathrm{m}$, and $\Delta n=$ $0.090-0.0029\left(T-T_{c}\right)$. Note that these values of the thickness and of the birefringence at the compensation point are very close to the expected ones: $25 \mu \mathrm{m}$ for the thickness and 0.09 for the birefringence $[14,15]$. In the following, we took for the thickness the value given by the fit.

Once the compensation temperature and the thickness of a sample were measured, we systematically recorded its transmittance curves for different temperature ramps. Two curves measured for two successive ramps of opposite signs are shown in Fig. 7. As expected the curve obtained by cooling is shifted to the left (towards lower temperatures), while the other obtained by heating is shifted to the right (towards higher temperatures). From these curves, we measured temperatures $T_{0}(r)$ by fitting the corresponding minima of the transmittance curve with parabolas (see in 


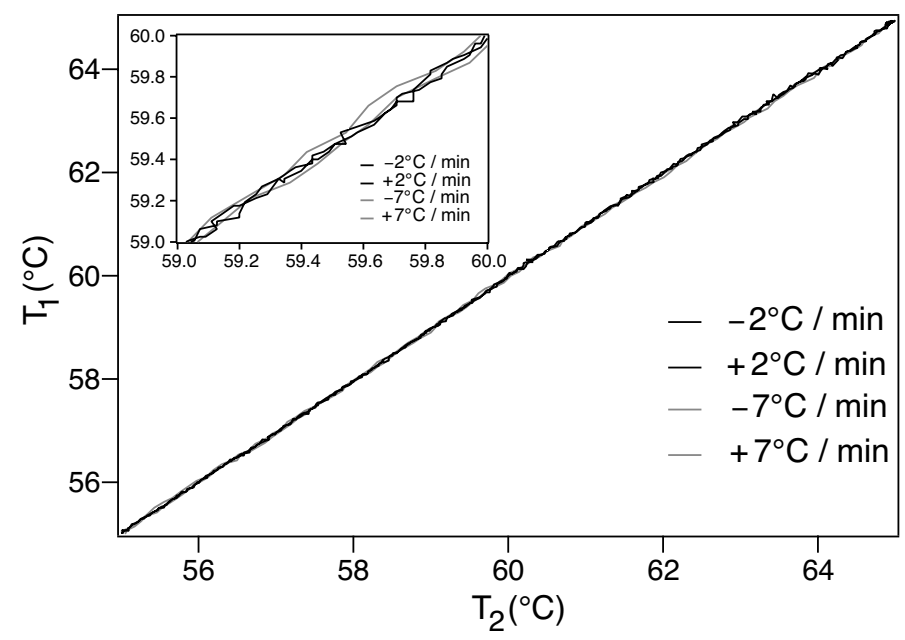

FIG. 5: Temperature of the side wire as a function of the temperature of the middle wire. The curves measured at $\pm 2^{\circ} \mathrm{C} / \mathrm{min}$ are superimposed (within the experimental noise) whereas those measured at $\pm 7^{\circ} \mathrm{C} / \mathrm{min}$ are systematically shifted by about $0.1^{\circ} \mathrm{C}$. Note that these curves are noisier than in Fig. 4 because of the use of the scanner card.

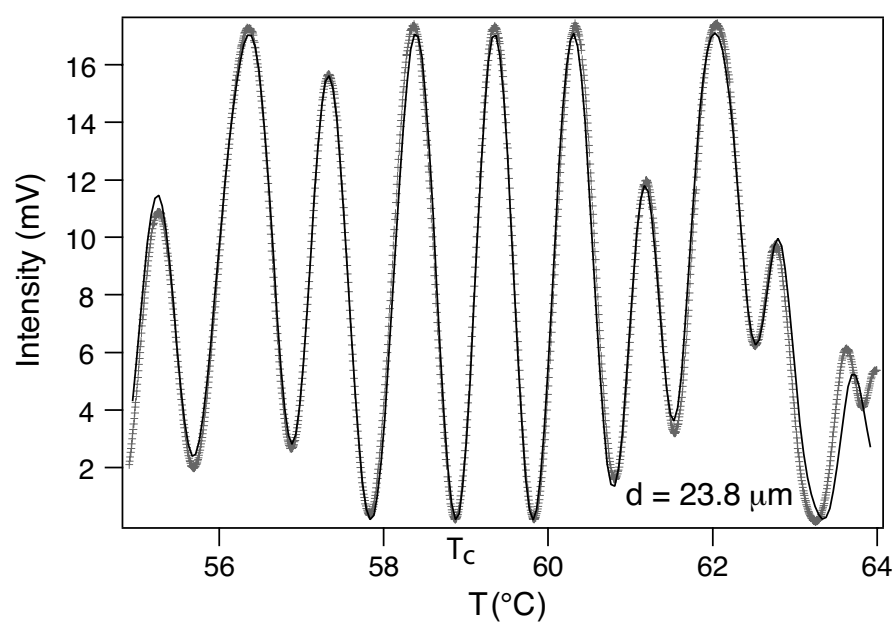

FIG. 6: Optical transmittance between crossed polarizers as a function of temperature. The temperature ramp was $0.2^{\circ} \mathrm{C} / \mathrm{min}$. Points are experimental and the solid line is the best fit to Eq. 12 .

Fig. 7). Curve $T_{0}(r)$ is shown in Fig. 8. It is well fitted by a linear law the slope of which gives the researched quantity $d T_{0} / d r$ defined in Eq.11. The experiment was then repeated for samples of different thicknesses. Our results are given in the next subsection. 


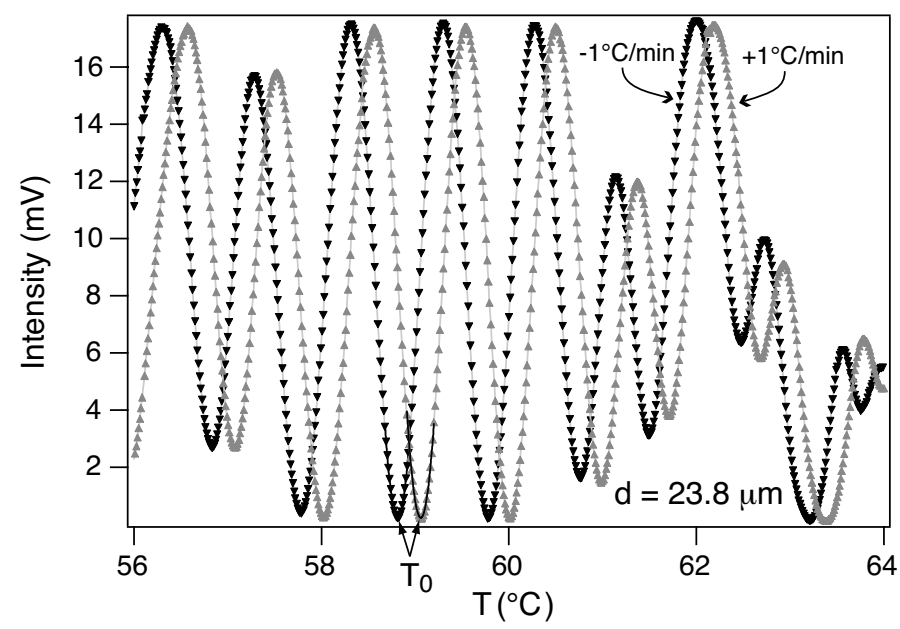

FIG. 7: Curves of optical transmittance measured between crossed polarizers as a function of temperature for two ramps of equal amplitude, but opposite signs. Temperatures $T_{0}$ are obtained by fitting with parabolas (solid lines) the local minima.

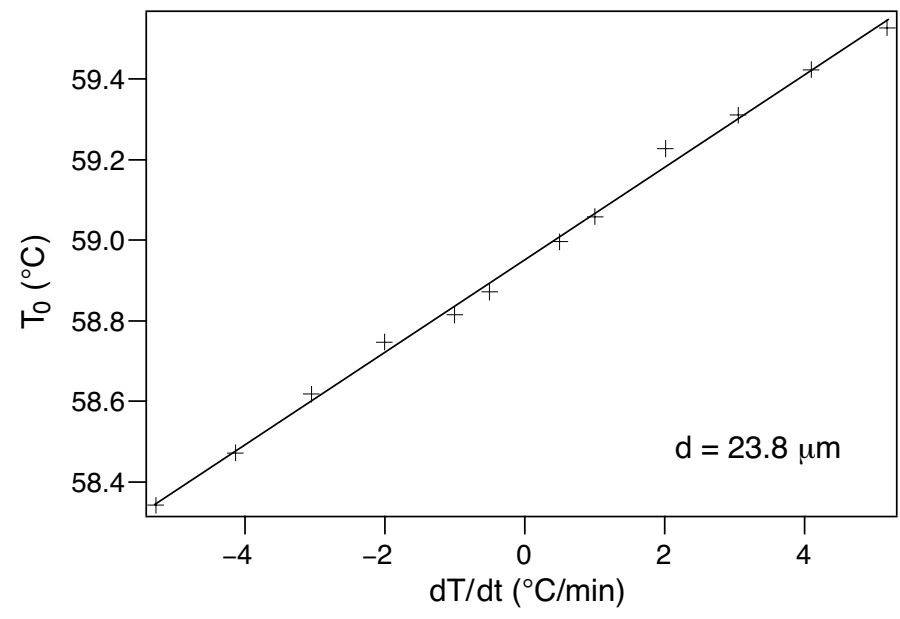

FIG. 8: Temperature $T_{0}$ as a function of the temperature ramp $r$ for a sample of a given thickness and its fit to a linear law.

\section{Results}

Fig. 9 collects all our experimental results obtained with samples of thicknesses ranging between 7 and $33 \mu \mathrm{m}$. In practice, it was not really possible to perform the experiment with thicker samples because of a spontaneous destabilization of the helical structure into cholesteric fingers when the temperature was changed (even as slowly as $\pm 1^{\circ} \mathrm{C} / \mathrm{min}$.

In order to find the value of the surface viscosity, we fitted this experimental curve to the theoretical law Eq. 11 by taking for the value of the bulk rotational viscosity $\gamma_{1}$ that measured previously: $0.075 \pm 0.009$ Pa.s and for the twist constant $K_{2}=2.8 \pm 0.2 \times 10^{-12} \mathrm{~N}[14,15]$. In this way, we found from the fit of the experimental data and by taking into account the uncertainties in the values of $\gamma_{1}$ and $K_{2}$ :

$$
\gamma_{S}=3.2 \pm 0.8 \times 10^{-7} \text { Pa.s.m }
$$

This value is discussed in the next section. 


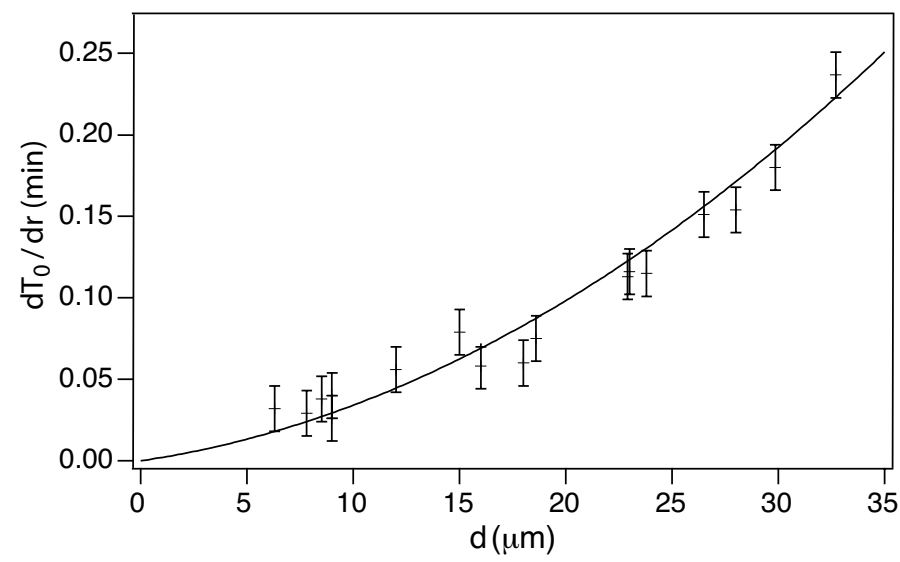

FIG. 9: Derivative $d T_{0} / d r$ as a function of the sample thickness $d$. The solid line is the best fit to Eq. 11 by taking $\gamma_{1}=0.075$ Pa.s.

\section{SIMPLE MODEL FOR THE SURFACE VISCOSITY}

This value of the surface viscosity is very large. Indeed, let us consider the viscosity $\eta$ of the polymercaptan hardener. Measurements in a rotating rheometer gave $\eta \sim 1 \mathrm{~Pa} . \mathrm{s}$ at the compensation temperature $\left(59^{\circ} \mathrm{C}\right)$ for shear rates ranging between 0.1 and $10 \mathrm{~s}^{-1}$. From these two quantities, we can form a length

$$
l_{S}=\frac{\gamma_{S}}{\eta}
$$

which turns out to be very large, of the order of $0.3 \mu \mathrm{m}$. This value is of the same order of magnitude as the thickness $l_{P}$ of the polymer layer we measured by Michelson interferometry.

This result thus suggests that the liquid crystal diffuses into the polymer layer over a typical distance $l_{D}$. This distance must be smaller than $l_{P}$ in order that the liquid crystal molecules do not adsorb on the glass (no memory effects were observed over many days on condition that the polymercaptan layer be thick enough [24]).

To estimate $l_{D}$, let us assume that each molecule dissolved in the polymer layer experiences a viscous torque $\Gamma_{m o l}$ proportional to its rotational velocity $\dot{\varphi}$. For a rod-like molecule of length $L$ and diameter $\Phi$, the viscous torque reads $[25]$

$$
\Gamma_{m o l}=\frac{\pi \eta L^{3} \dot{\varphi}}{3(\ln (L / \Phi)-0.8)}
$$

Let $n_{S}$ be the number of molecules dissolved in the polymer layer per unit surface area. In a crude model, the surface torque $\Gamma_{S}$ introduced phenomenologically in Eq. 1 reads:

$$
\Gamma_{S} \sim n_{S} \Gamma_{m o l}
$$

which gives the surface viscosity

$$
\gamma_{S} \sim n_{S} \frac{\pi \eta L^{3}}{3(\ln (L / \Phi)-0.8)}
$$

Finally, writing that $l_{D} \sim n_{S} L \Phi^{2}$, we obtain from the preceding equation

$$
l_{D} \sim \frac{\gamma_{S}}{\eta}\left(\frac{\Phi}{L}\right)^{2}\left[\ln \left(\frac{L}{\Phi}\right)-0.8\right]
$$


In practice $L \sim 30 \AA, \Phi \sim 5 \AA$ which gives from the measured value of $\gamma_{S}$ and $\eta$ given before: $l_{D} \sim 0.01 \mu \mathrm{m}=100 \AA$. As expected, we find that $l_{D}$ is smaller than the thickness of the polymer layer, although this calculation obviously underestimates its value.

To summarize, the large value of $l_{S}$ defined in Eq. 13 is certainly a clear indication that the nematic liquid crystal dissolves partially in the polymer surface layer. On the other hand, $l_{D}$ must be smaller than the thickness of the polymer layer in order to prevent that the liquid crystal molecules adsorb on the glass. These conclusions are very similar to those drawn before by Vilfan et al [26] who also measured by dynamic light scattering very large values of $l_{S}$ for the liquid crystal $5 \mathrm{CB}$ in contact with a photoaligning poly-(vinyl-cinnamate) layer.

\section{ANNIHILATION TIME OF TWO DISCLINATION LINES OF OPPOSITE SIGNS}

Before concluding the article, let us return to the problem of the annihilation of the two $\pm 1 / 2$ disclination lines shown in Fig. 1b. Indeed, their annihilation may seem too long as it takes about 11.5 min while the initial distance between the two defects is of "only" $110 \mu \mathrm{m}$. Thus, one may wonder whether this observation is compatible with a fully sliding anchoring.

To show it is indeed the case, let us estimate this time. A straightforward calculation neglecting the backflow effects and the material elastic anisotropy gives:

$$
t_{\text {annihil }}=\frac{\gamma_{1}^{\star}}{4 K} r_{0}^{2} \ln \frac{r_{0}}{r_{c}}
$$

where $r_{0}$ is the initial distance between the two defects, $r_{c}$ a core radius of molecular size, $K=\frac{K_{1}+K_{3}}{2}$ the average of the splay and bend constants and $\gamma_{1}^{\star}$ an effective viscosity taking into account the surface viscosity: $\gamma_{1}^{\star}=\gamma_{1}+2 \gamma_{S} / d$. With the experimental values $d=10 \mu \mathrm{m}, r_{0}=110 \mu \mathrm{m}, K=4.6 \times 10^{-12} \mathrm{~N}[14,15], \gamma_{1}=0.075 \mathrm{~Pa} . \mathrm{s}$ and $\gamma_{S}=$ $3.2 \times 10^{-7}$ Pa.s.m, we calculate $t_{\text {annihil }} \approx 16 \mathrm{~min}$ by taking $r_{c} \approx 50 \AA$. This time is longer than that observed experimentally $(11.5 \mathrm{~min})$. This difference is nevertheless not surprising as we know from numerical simulations that backflow effects tend to reduce by a typical factor of 1.5 the annihilation time between the two defects [18, 20]. This interpretation is reinforced by the important experimental fact that the $+1 / 2$ defect moves faster than the $-1 / 2$ defect, another phenomenon predicted by the theory [18-20]. We thus conclude that our observations on defect annihilation are compatible with a sliding anchoring of the molecules on the polymer layer.

\section{CONCLUSION}

We have described a new surface treatment which allows us to obtain a sliding anchoring of the molecules of a compensated cholesteric mixture. We checked experimentally that this anchoring was efficient for other liquid crystals such as 5CB and 8CB, but we did not performed systematic measurements with these materials. By using the large variation of the cholesteric pitch around the compensation point, we were able to measure the surface viscosity of the liquid crystal. We emphasize that this experiment was made possible owing to the development of temperature sensors able to measure in-situ the sample temperature. We have found that the surface viscosity $\gamma_{S}$ was large, which we interpreted by assuming that the liquid crystal diffuses within the polymer layer over a characteristic length $l_{D}$ much larger than a molecular length. This contrasts with usual surface treatments (as, for instance, bare glass or covered with a $\mathrm{SiO}$ or a polymerized PVA or PI layer) where $l_{D}$ or more precisely $l_{S}$ (defined in this case as the ratio $\left.\gamma_{S} / \gamma_{1}\right)$ is of the order of a molecular length [27]. In the latter case, the origin of the surface viscosity is certainly very different and was explained as due a backflow effect close to the surface [28]. Nevertheless this explanation cannot 
apply here as we are dealing with azimuthal (and not zenithal) molecular rotations. As a consequence, we only have twist deformations which do not generate any backflow. Finally, note that all our experiments were performed in the stationary regime (i.e., at times $t \gg 1 / \omega_{b}$ and $1 / \omega_{S}$ ), which allowed us to escape the complications caused by the incompatibility (at the initial time, when the ramp is started) between the bulk equation (2) and the surface equation (4) $[29]$

One of our objective in the future will be to improve the model of the surface viscosity. This could be done by using a tensorial description [30] of the Ericksen-Leslie theory of the nematohydrodynamics coupled with a Cahn-Hilliard description [31] of the nematic-polymer interface [32]. It would be also interesting for applications to better determine the zenithal anchoring energy, for instance, by measuring the saturation voltage above which the director is fully realigned by the field. Another crucial point for applications would be to study aging properties of this new anchoring over longer period of time than two or three days in order to check whether memory effects develop at long time. Such studies are planed in the future, in particular as a function of the thickness of the polymercaptan layer.

\section{Acknowledgments}

This work has been supported by the Polonium Program N¹1622QC.

\section{APPENDIX}

In our experiments, the average sample temperature changes quickly during the steepest ramps. As a consequence the temperature can no longer be considered as constant along the $z$ axis. In these conditions Eq. 2 is only approximate because it neglects the spatial variations of $K_{2}$ and $q$. As in practice $K_{2}$ changes much less rapidly than $q$, we shall only consider the $q$ variation assuming $K_{2}=$ constant. In these conditions, a new term proportional to $\partial q / \partial z$ occurs in the bulk torque equation (2) which becomes

$$
\gamma_{1} \frac{\partial \varphi}{\partial t}=K_{2} \frac{\partial^{2} \varphi}{\partial z^{2}}-K_{2} \frac{\partial q}{\partial z}
$$

In order to determine whether the new term $-K_{2} \frac{d q}{d z}$ is pertinent, let us calculate its order of magnitude with respect to the two others by using the simplified solution given by Eq. 10. By denoting by $\Delta T$ the typical temperature variation across the sample thickness $d$, we find:

$$
\gamma_{1} \frac{\partial \varphi}{\partial t}=\gamma_{1} \omega=\gamma_{1} d \frac{d q}{d T} \frac{\partial T}{\partial t}
$$

while

$$
K_{2} \frac{\partial q}{\partial z}=K_{2} \frac{d q}{d T} \frac{\partial T}{\partial z} \approx K_{2} \frac{d q}{d T} \frac{\Delta T}{d}
$$

Inside the sample, the temperature satisfies the heat equation

$$
\frac{\partial T}{\partial t}=D_{T} \frac{\partial^{2} T}{\partial z^{2}}
$$

where $D_{T}$ is the thermal diffusivity of the liquid crystal. From this equation, we find that in order of magnitude

$$
\frac{\partial T}{\partial t}=D_{T} \frac{\Delta T}{d^{2}}
$$


This allows us to calculate the ratio between the two terms:

$$
\frac{\gamma_{1} \frac{\partial \varphi}{\partial t}}{K_{2} \frac{\partial q}{\partial z}} \approx \frac{\gamma_{1} d \frac{d q}{d T} D_{T} \frac{\Delta T}{d^{2}}}{K_{2} \frac{d q}{d T} \frac{\Delta T}{d}}=\frac{D_{T}}{D_{o}}
$$

where $D_{o}=\frac{K_{2}}{\gamma_{1}}$ is the orientational diffusivity. In our sample, $D_{o} \approx 3.7 \times 10^{-11} \mathrm{~m}^{2} \mathrm{~s}^{-1}$. As for its thermal diffusivity, it must be of the same order of magnitude as in usual nematics: $D_{T} \approx 10^{-7} \mathrm{~m}^{2} \mathrm{~s}^{-1}[33$, 34]. From these two values we can estimate that

$$
\frac{\gamma_{1} \frac{\partial \varphi}{\partial t}}{K_{2} \frac{d q}{d z}} \approx 3000
$$

This fully justifies neglecting the term in $\frac{d q}{d z}$ in the bulk torque equation.

[1] P. Oswald and P. Pieranski, Nematic and Cholesteric Liquid Crystals: Concepts and Physical Properties Illustrated by Experiments (Taylor \& Francis, CRC press, Boca Raton 2005).

[2] B. Jérôme, Rep. Prog. Phys. 54, 391 (1991).

[3] B. Jérôme, P. Pieranski, and M. Boix, EuroPhys. Lett. 5, 693 (1988).

[4] J. Bechhoefer, B. Jérôme, and P. Pieranski, Phys. Rev. A 41, 3187 (1990).

[5] G. P. Bryan-Brown, E. L. Wood, and I. C. Sage, Nature 399, 338 (1999).

[6] I. Dozov, D. N. Stoenescu, S. Lamarque-Forget, P. Martinot-Lagarde, and E. Polossat, Appl. Phys. Lett. 77, 4124 (2000).

[7] O. Ou Ramdane, P. Auroy, S. Forget, E. Raspaud, P. Martinot-Lagarde, and I. Dozov, Phys. Rev. Lett. 84, 3871 (2000).

[8] M. Nespoulos, C. Blanc, and M. Nobili, J. Appl. Phys. 102, 073519 (2007).

[9] I. Dozov, M. Nobili, and G. Durand, Appl. Phys. Lett. 70, 1179 (1997).

[10] L. Komitov, J. Yamamoto, and H. Yokoyama, J. Appl. Phys. 89, 7730 (2001).

[11] L. Komitov, B. Helgee, J. Felix, and A. Matharu, Appl. Phys. Lett. 86, 023502 (2005).

[12] D. N. Stoenescu, H. T. Nguyen, P. Barois, L. Navailles, M. Nobili, P. Martinot-Lagarde, and I. Dozov, Mol. Cryst. Liq. Cryst. 358, 275 (2001).

[13] C. Blanc, D. Svenšek, S. Žumer, and M. Nobili, Phys. Rev. Lett. 95, 097802 (2005).

[14] A. Dequidt and P. Oswald, EuroPhys. Lett. 80, 26001 (2007).

[15] A. Dequidt, A. Żywociński, and P. Oswald, Lehmann effect in a compensated cholesteric liquid crystal: experimental evidence with fixed and gliding boundary conditions, submitted to Eur. Phys. J. E.

[16] We would like to emphasize that the decrease of $3-4{ }^{\circ} \mathrm{C}$ of the melting temperature reported in Ref. [15] was mainly due to a pollution of the liquid crystal by the ketone.

[17] P. Oswald and J. Ignés-Mullol, Phy. Rev. Lett. 95, 027801 (2005).

[18] G. Toth, C. Denniston, and J. M. Yeomans, Phy. Rev. Lett. 88, 105504 (2002).

[19] E. I. Kats, V. Lebedev, and S. V. Malinin, J. Theo. Exp. Phys. 95, 714 (2002).

[20] D. Svenšek and S. Žumer, Phy. Rev. Lett. 90, 155501 (2003).

[21] J. Nehring, A. R. Kmetz, and T. J. Scheffer, J. Appl. Phys. 47, 850 (1976).

[22] A. Jǎkli, D. R. Kim, M. R. Kuzma, and A. Saupe, Mol. Cryst. Liq. Cryst. 198, 331 (1991).

[23] P. G. de Gennes, The Physics of Liquid Crystals (Clarendon Press, Oxford 1974), p.97.

[24] Samples prepared by using a concentration of $2 \%$ in mass of hardener in the ketone showed clear memory effects after one day at the compensation temperature. In this case, the planar anchoring was memorized on the plate treated for gliding anchoring and a weak azimuthal anchoring energy was occurring as in Ref. [8].

[25] R. G. Larson, The Structure and Rheology of Complex Fluids (Oxford University Press, Oxford, 1999).

[26] M. Vilfan, I. Drevenšek Olenik, A. Mertelj, and M. Čopič, Phys. Rev. E. 63, 061709 (2001).

[27] A. Mertelj and M. Čopič, Phys. Rev. E. 61, 1522 (2000).

[28] G. E. Durand and E. G. Virga, Phys. Rev. E. 59, 4137 (1999).

[29] A. M. Sonnet, E. G. Virga, and G. E. Durand, Phys. Rev. E. 62, 3694 (2000).

[30] T. Qian and P. Sheng, Phys. Rev. E. 58, 7475 (1998).

[31] J. W. Cahn, Trans. Metall. Soc. AIME 242, 166 (1968).

[32] V. Popa-Nita and P. Oswald, J. Chem. Phys. 127, 104702 (2007).

[33] W. Urbach, H. Hervet, and F. Rondelez, Mol. Cryst. Liq. Cryst. 46, 209 (1978).

[34] G. Ahlers, D. S. Cannell, L. I. Berge, and S. Sakurai, Phys. Rev. E 49, 545 (1994). 